\title{
Über die Chromosomenzahl der zwei Arten von japanischen Austern
}

\author{
Haruo Kobayashi \\ Biologisches Institut, Minami Zweigschule, \\ Hiroshima Universitat, Jaran
}

Eingegangen am ‥ Juni 1954

Die Chromosomenzahl einer Art der Austern, Ostrea laperousi, wurde schon vom Verfasser (1952) karyologisch untersucht, wobei er ihrer $2 n=24$ angegeben hat. Mit den heutigen Studien wird es versucht, die haploide Zahl derselben Arten zu bestimmen, und die diploide Zahl derselben Arten aus Hiroshima mit denjenigen aus Ariake und aus Sendai zu verglichen. Dazu wird hier auch die diploide Zahl der anderen Art der Austern beschrieben.

Die Arbeit wird vom Verfasser mit der wirksamen Hilfe der Prof. Y. ABE und Prof. S. HAREYAMA an der Universität Hiroshima ausgeführt, denen ich hiermit meinen allerbesten Dank aussprechen möchte. Auch für Unterstützung beim Beschaffen der Materialien sagt der Verfasser hier dem Personal der Versuchsstationen für Fischerei Miyagi und Ariake seinen herzlichen Dank.

\section{Materialien und Methode}

Die Materialien, die dem Verfasser zur Verfügung stehen, sind Ostrea laperousi SCHRENCK, einige die wild gewachsene aus dem Flusse Ohta an der Bai von Hiroshima in der Stadt Hiroshima, und andere die gezüchtete

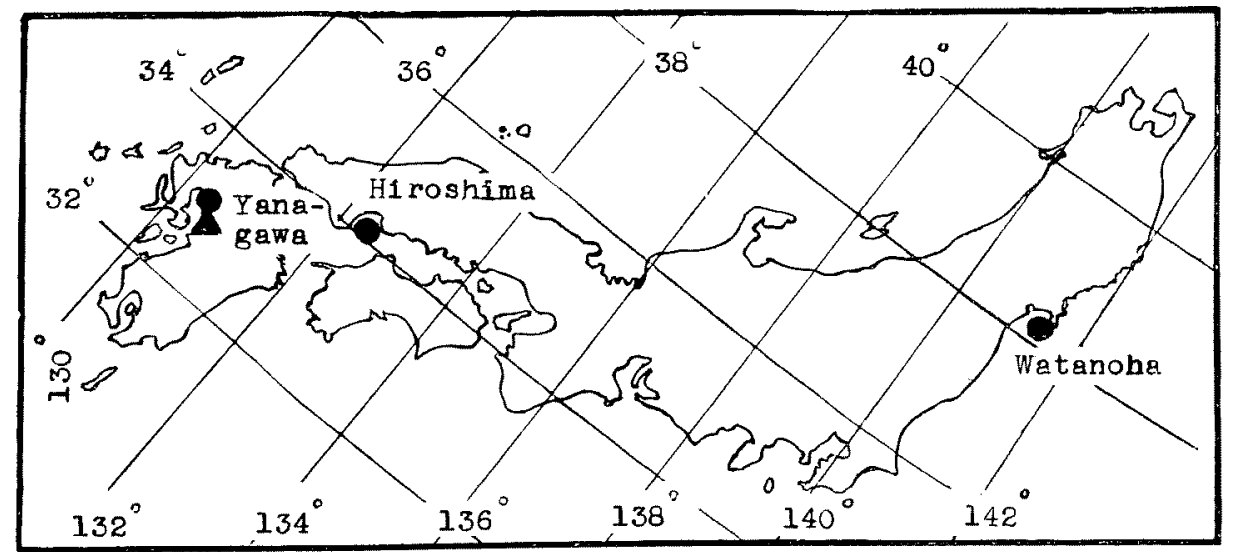

Abb. 1. Die Orte, wovon die Austern gesammelt wurden.

- Ostrea laperousi Schrenck. A O. ariakensis Wakiya.

aus Watanoha an der Bai von Sendai in der Provinz Miyagi und aus Yanagawa an der Bai von Ariake in der Provinz Fukuoka, und auch noch die gezüchtete Ostrea ariakensis WAKIYA aus Yanagawa (Abb. 1). 
Modifizierte NAWASCHIN'sche Flüssigkeit wird als Fixierungsmittel benützt. Zur Untersuchung der Gonien-Chromosomen wurden die Materialien $10 \mu$ dünn zerschneidet, und für die Beobachtung der heterotypische Teilung die künstlich befruchteten Eier $15 \mu$ dünn zerchneidet, dann einige Materialien mit Hämatoxylin nach HEIDENHAIN und andere mit Safranin und Indigo-Carmin nach BORREL gefärbt, wie es schon von KOBAYASHI (1954) berichtet.

\section{Beobachtung}

A) Ostrea ariakensis WAKIYA (Abb. 2)

Die Chromosomenzahl in den Oogonien von Ostrea ariakensis WAKIYA ist $2 n=24$. Unter diesem Chromosomenbestand gibt es 22 der V-förmigen und 2 der kleinsten stäbchenförmigen Chromosomen. Durch nähere Vergleichung der Chromosomen aus beiden Arten von $O$. ariakensis und O. laperousi können wir feststellen, daß sich die Chromosomen der ersteren hauptsächlich

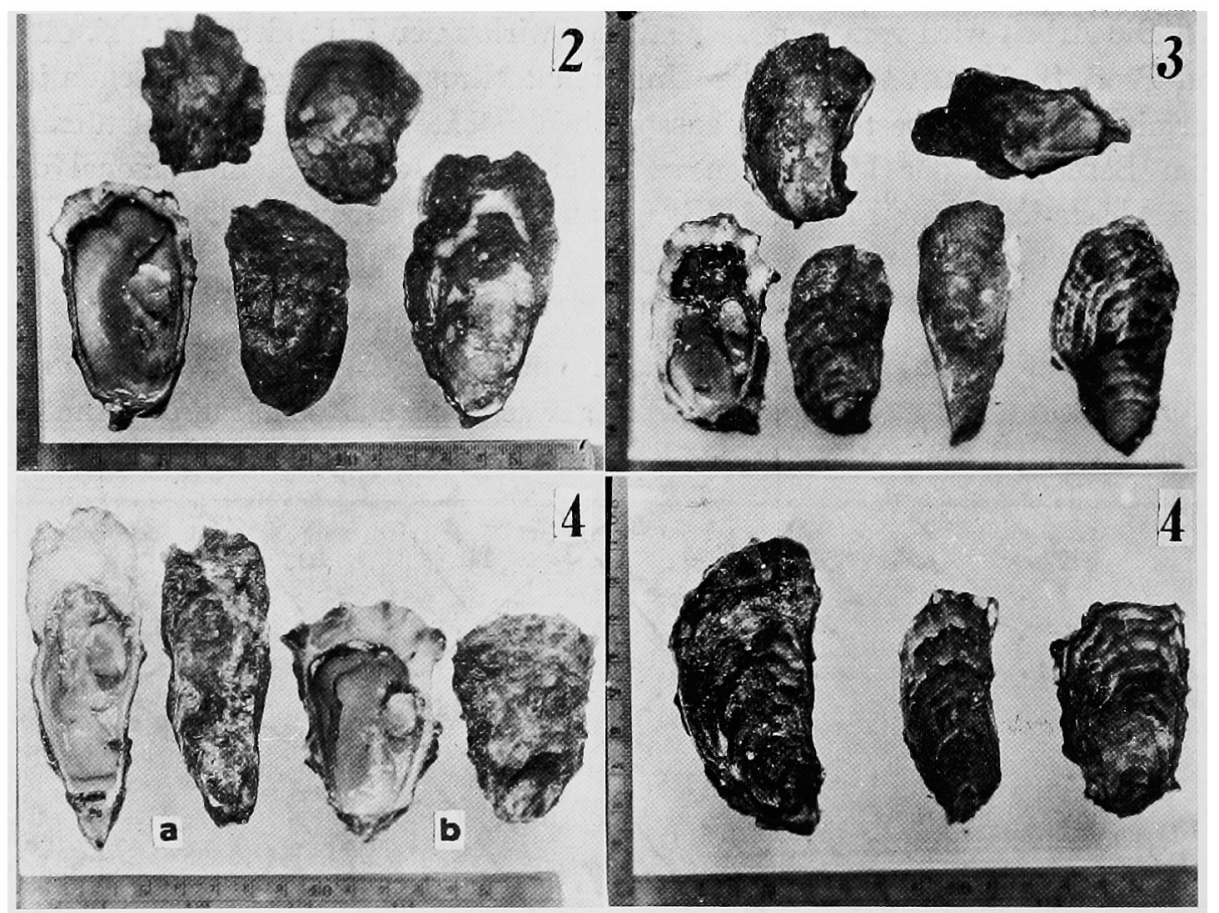

Abb. 2-4. 2. Ostrea arialensis WAKIYA in der Bai von Ariake. 3. Ostrea laperousi Schrenck in der Bai von Ariake. 4. Ostrea laperousi Schrenck in der Bai von Sendai. a eine lange Form, b eine kurze Form.

in den folgenden drei Punkten von der letzteren unterscheiden: (1) die Spitzen der V-förmigen Chromosomen von ariakensis sind viel dünner als dieselben von laperousi, (2) die kleinsten Chromosomen von ariakensis sind nicht V-: sondern stäbchenförmig, und (3) bei ariakensis werden keine L-förmige, riesig und mittelgroß, gefunden. Aber in Betreff anderer Merkmalen der Chromo- 
somen, sind $O$. ariakensis und $O$. laperousi ganz dieselben, wie Abb. 5 und 6 . B) Ostrea laperousi Schrenck (Abb. 3 und 4)

Es gibt zwei Formen der Ostrea laperousi: die eine ist kurz und die andere lang. Die lange Form ist bezüglich ihrer Gestalt der $O$. gigas THUNBERG sehr ähnlich, d.h. die Muschel ist $9.8 \mathrm{~cm}$. lang, an ihrem Herzen $3.2 \mathrm{~cm}$. breit und $2.5 \mathrm{~cm}$. hoch; dagegen ist die kurze Form $6.8 \mathrm{~cm}$. lang, $4 \mathrm{~cm}$. breit und $2 \mathrm{~cm}$. hoch.

a) Die kurze Form kommt erst bei meinen Studien zur Verwendung. Es gibt keine bemerkbaren Verschiedenheiten über die Karyotypen von Austern, wenn der Verfasser $O$. laperousi aus Hiroshima mit denen aus Sendai und - aus Ariake einander vergleicht, wobei selbstverständlich die Austern der ähnlichen Gestalten zum Vergleich heranzuziehen sind.

Ostrea laperousi aus Sendai und aus Ariake haben sowohl 24 Chromosomen an diploider Zahl und dieselbe Gestalten der Chromosomen wie die aus Hiroshima: die meisten Chromosomen sind V-förmig, aber die Spindelfasern haften unfehlbar an den zweitlängsten Chromosomen und der mittelgroßen Chromosomen, gleichfalls an ihrem Submittel $z$ u den L-förmigen zu werden, was in Tafel 1 und Abb. 7, 8 und 9 lautet.

Tafel 1. Chromosomenzahl der Austern

\begin{tabular}{|c|c|c|c|c|c|}
\hline Species & Heimat & Oogronium & $\begin{array}{l}2 \mathrm{n} \\
\text { Spermatogonium }\end{array}$ & Karyotypus & $\mathrm{n}$ \\
\hline O. ariakensis & Ariake & 24 & - & $22 V+2$ Stäb. & - \\
\hline $\begin{array}{l}\text { O. laperousi } \\
\text { (kurze Form) }\end{array}$ & Hiroshima & 24 & 24 & $4 \mathrm{~L}+20 \mathrm{~V}$ & 12 \\
\hline " & Ariake & - & 24 & $4 \mathrm{~L}+20 \mathrm{~V}$ & - \\
\hline$"$ & Sendai & 24 & 24 & $4 \mathrm{~L}+20 \mathrm{~V}$ & - \\
\hline (lange Form) & Sendai & 24 & - & $4 \mathrm{I}+20 \mathrm{~V}$ & 一 \\
\hline
\end{tabular}

Die haploide Chromosomenzahl läßt sich mit der O. laperousi an der Bai von Hiroshima bestimmen. Da die künstlich befruchteten Eier eben im Begriffe sein die ersten Polzellen hervorzubringen, ist die haploide Zahl leicht zu beobachten. Es gibt 12 Tetraden in der Metaphase der ersten Oozyten (Abb. 13, 14, 15 u. 18). Die allgemeinen Gestalten der Tetraden sind kugelförmig und darunter bemerken wir 8 grösse und 4 kleine Chromosomen. Diese 12 Tetraden teilen sich in zwei Gruppen, jede von beiden mit 12 Bivalenzen konstituiert d.h. die kugelförmige Tetraden sind zu den V-förmigen Bivalenzen in der zweiten Oozyten geworden (Abb. $16 \mathrm{u.} \mathrm{17).}$

Keine Geschlechtschromosomen kommen zur Beobachtung auch im Vergleich mit der Autosomen in Bezug auf die Gestalt, die Bewegung und die Farbenabstufung während der Meiosis.

Es kann selten noch der Fall eintreten; wo ein künstlich befruchtetes Ei 48 Chromosomen enthält, doch der Durchmesser dieser Eizellen ist ca. $52 \mu$ 

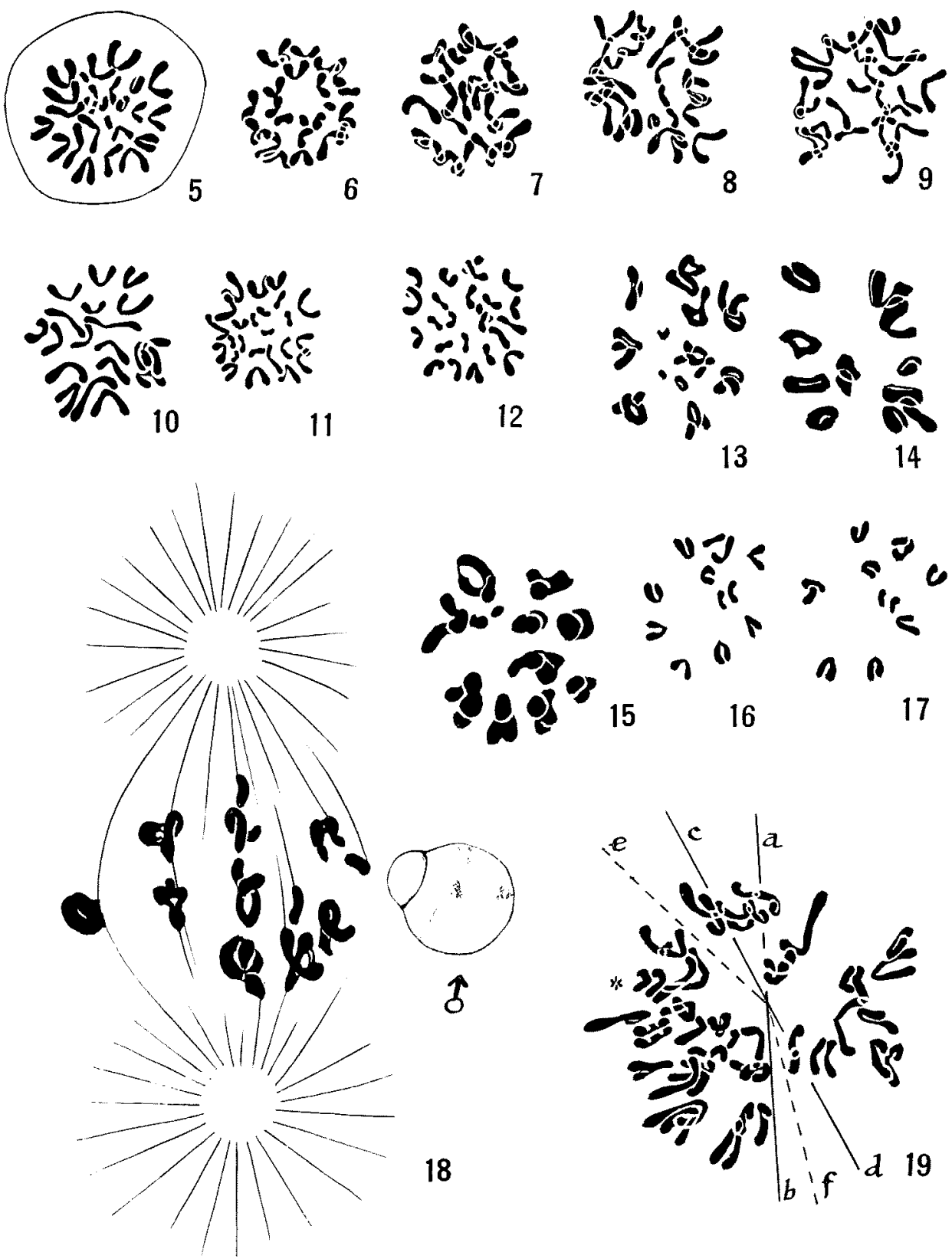

Abb. 5-19. 5u. 6. Oogonien von Ostrea arialensis Wakry . $2 n=24=22 \mathrm{~V}+2$ Stäb. 7. Spermatogonium von Ostrea laperousi SCHRENCK in der Bai von Ariake. $2 n=24=4 \mathrm{~L}$ $+20 \mathrm{~V}$. 8. Spermatogonium von Ostrea laperousi Schrenck in der Bai von Sendai. $2 n=24=4 \mathrm{~L}+20 \mathrm{~V}$. 9. Oogonium von Ostrea laperousi SCHRENCK in der Bai von Sendai. $2_{n}=24=4 \mathrm{~L}+20 \mathrm{~V}$. 10. Oogonium von Ostrea laperousi, die lange Form in der Bai von Sendai. Eine Anzahl Chromosomen verlieren sich beim Schneiden. 11 u. 12. Ibidem. $2 n=24=4 \mathrm{~L}+20 \mathrm{~V} . \quad 13,14 \mathrm{u}$. 15. Erste Oozyten von Ostrea laperousi in der Bai von Hiroshima. 12 Tetraden werden erkannt. $16 \mathrm{u} .17 .12$ Tetraden teilen sich in zwei 
und sie ist so groß wie die gewöhnlichen Eiern. Die Spindelachsen bei der Teilung schlagen zwei Richtungen mit der Winkel ca. $30^{\circ}$ ein und die Kernplatte befindet sich fast in der Mitte der Eizellen (Abb. 19). Gleichgroße und gestaltete Chromosomen liegen näher miteinander, auch die Formen jeder Chromosomen sind denen der Gonienzellen gleich. Daraus erklären sich die Kernplatten in diesen Eizellen so: diese zeigt nicht eine tetraploide Zelle sonderen auch eine diploide, und beide Kerne von Eizelle und von Spermium vereinigen sich auf denselben Kernplatten, dann die Befruchtung im engeren Sinne hat vollendet, und eben jetzt der erste Furchung der Zygote angefangen. Auch bei dieser Zygote zeigt es sich, daß die diploide Chromosomen von O. laperousi 24 zählen.

b) Dann glücklicherweise gelingt es dem Verfasser, die diploide Chromosomenzahl der langen Formen von $O$. laperousi aus Sendai klar zumachen (Abb. 4 a). Die Oogonium hat 24 Chromosomen, d.h. 4 L- und 20 V-Formen, wie es in den kurzen Formen im allgemeinen beobachtet wird. (Abb. 10, 11 und 12). Vergleichen wir die langen Formen der O. laperousi mit den kurzen Formen in Bezug auf die Chromosomenzahl, ihre Gestalt und Größe und die Stelle der Kinetochore, so können wir die beiden Formen deutlich und genau unterscheiden nicht.

\section{Zusammenfassung}

1. Die Chromosomenzahl von Ostrea ariakensis WAKIYA beträgt $2 n=24$, bei der Metaphase der Oogonien. Ein Paar der kleinsten Chromosomen stäbchenförmig und andere Paaren V-förming, also ihr Karyotypus wird folgendermassen ausgedrückt. $24=22 \mathrm{~V}+2$ Stäb.

2. Die Chromosomenzahl von gewöhnlichen Ostrea laperousi SCHRENCK an den Baien von Hiroshima, Sendai und Ariake ist $2 n=24$, wobei darunter 4 die L-förmigen und 20 die $\mathrm{V}$-förmigen darstellen.

3. O. laperousi aus verschiedenen Heimaten stimme bezüglich der Chromosomenzahl und -formen völlig überein, und kein anderer Typus der $O$. laperousi von karyologischem Standpunkte aus wird bemerkt.

4. Die haploide Zahl von $O$. laperousi beträgt $n=12$, bei der Metaphase der ersten Teilung, die nach der künstlichen Befruchtung erkannt wird.

Gruppen der 12 Bivalenzen. Die Chromosomen lauteten in Abb. 16 soll nach der ersten Polzelle gehen, und die Chromosomen in Abb. 17 soll zu der zweiten Oozyte bleiben. 18. Eine riesige Kernplatte der ersten Oozyte mit einem Spermium, die Tetraden sehen schräg an. 19. Eine riesige Kernplatte der Zygotzelle. Zwei Spindelachsen ab and ed mit der Winkel ca. $30^{\circ}$, und die Chromosomen läßt sich in zwei Gruppen bei Linie ef teilen, dabei 2 Chromosomen mit * gehören zu der rechten Gruppe. Die Achse ab muB senkrecht gegen dieser Papierflache stehen, aber in dieser Abbildung wird die Achse ab nach der Richtung projiziert, daß sich die Achse ab $90^{\circ} \mathrm{um}$ die Achse cd dreht.

Alle Chromosomen wurden mit Hämatoxylín nach HEIDENHAIN gefärbt, nur die von Abb. 11 und 12 mit Safranin und Indigo-Carmin gefärbt. Vergrößerung $3800 \times 2 / 3$. 
5. Die lange Form der O. laperousi ist bezüglich des Chromosomenbestandes mit der kurzen Form ganz ähnlich.

\section{Schrifttum}

Kobayashi, H. 1952. On the chromosome number in the oyster (Ostrea laperousi Schrenck). Jour. Sci. Hiroshima Univ. Ser. B. Div. 1. 13: 53-59.

- 1954. Cyto-chemical study on the nucleolus in the common Japanese oyster (Ostrea laperousi SChRENCK), II. Nucleic acids and nucleoproteins. Ibidem 15: 167180.

Cy to $\log$ i a vol. 19, no. 4 (pp. 255-376)

Issued December 30, 1954

Ausgegeben am 30. Dezember 1954

Paru le 30 Decembre 1954 